\title{
A Closed-Form Formulation for the Build-Up Factor and Absorbed Energy for Photons and Electrons in the Compton Energy Range in Cartesian Geometry
}

\author{
Volnei Borges, Julio Cesar Lombaldo Fernandes, Bardo Ernest Bodmann, Marco Túllio Vilhena, \\ Bárbara Denicol do Amaral Rodriguez \\ Department of Applied Math, Universidade Federal do Rio Grande do Sul, Porto Alegre, Brazil \\ Email: \{borges, julio.lombaldo, bardo.bodmann, vilhena\}@ufrgs.br
}

Received October 5, 2011; revised November 26, 2011 ; accepted December 8, 2011

\begin{abstract}
In this work, we report on a closed-form formulation for the build-up factor and absorbed energy, in one and two dimensional Cartesian geometry for photons and electrons, in the Compton energy range. For the one-dimensional case we use the $L T S_{N}$ method, assuming the Klein-Nishina scattering kernel for the determination of the angular radiation intensity for photons. We apply the two-dimensional $L T S_{N}$ nodal solution for the averaged angular radiation evaluation for the two-dimensional case, using the Klein-Nishina kernel for photons and the Compton kernel for electrons. From the angular radiation intensity we construct a closed-form solution for the build-up factor and evaluate the absorbed energy. We present numerical simulations and comparisons against results from the literature.
\end{abstract}

Keywords: Build-Up Factor; Compton Energy; Cartesian Geometry; Fokker-Plank Equation

\section{Introduction}

In radiological protection the effectiveness of a material as a biological shield is related to its cross-section for scattering and absorption which is cast into physical parameters. The determination of those parameters require the solution of a systems of linear transport equations, i.e. the fluence for photons and electrons. Established methods that solve the transport equations are the PN approximation [1], the discrete ordinate method and their variants [2]. The $S_{N}$ method has been used successfully in photon transport calculation, whereas recently the $P_{N}$ approximation was applied to electron transport. In this work we present the solution of a couple system of linear transport equations, using the $L T S_{N}$ method for a rectangular domain considering the Klein-Nishina scattering kernel and a multi-group model for photons. The electron contribution to energy deposition induced by incident photons is quantified solving the two-dimensional Fokker-Planck equation for electron transport $[3,4]$ by the $P_{N}$ approximation in the angular variable followed by applying the Laplace Transform to one of the spatial variables (here $x$ ). This procedure leads to a closed-form formulation for the build-up factor and absorbed energy, in one and two dimensional Cartesian geometry for photons and electrons, in the energy range where Compton scattering is dominant [5].

\section{The $L T S_{N}$ Nodal Solution in Two Dimensional for Photons}

The two-dimensional $S_{N}$ nodal problem for photons, assuming Klein-Nishina scattering kernel and a multi-group model is

$$
\begin{aligned}
& \mu_{n} \frac{\partial}{\partial x} I_{j n}(x, y)+\eta_{n} \frac{\partial}{\partial y} I_{j n}(x, y)+\mu_{1 j} I_{j n}(x, y) \\
= & \frac{\Delta}{3} \sum_{l=0}^{L} \frac{2 l+1}{2} \sum_{r=1}^{G} c_{r} \alpha k_{r j} P_{l}\left(1+\lambda_{r}-\lambda_{j}\right) P_{l}\left(\mu_{n}\right) \\
& \times \sum_{i=1}^{N} P_{l}\left(\mu_{i}\right) I_{r i}(x, y) w_{i}
\end{aligned}
$$

subject to vacuum boundary conditions in a rectangle $0 \leq x \leq a$ and $0 \leq y \leq b$. Where $j=1, \cdots, G ; n=1, \cdots$, $N ; N=M(M+2) / 2$ is the cardinality of the discrete ordi- nate set, $M$ represents the order of the angular quadrature, $G$ is the number of energy groups in units of wavelengths, $\mu_{l j}$ is the linear attenuation coefficient, $I_{j n}(x, y)=I\left(x, y, \lambda_{j}, \Omega_{n}\right)$ is the angular flux into the discrete direction $\Omega_{n}=\left(\mu_{n}, \eta_{n}\right)$ for the $j$-th energy group, $w_{i}$ are the Lewis-Miller quadrature weights and

$k_{r j}=k\left(\lambda_{r}, \lambda_{j}\right)$ is the Klein-Nishina scattering kernel, defined as

$$
k_{r j}=\frac{3}{8} \frac{\lambda_{r}}{\lambda_{j}}\left(\frac{\lambda_{r}}{\lambda_{j}}+\frac{\lambda_{j}}{\lambda_{r}}-\sin ^{2} \sin ^{2} \theta\right)
$$

The $L T S_{N}$ nodal approach for photons yields the following $S_{N}$ equation system 


$$
\begin{aligned}
& \eta_{n} \frac{\partial}{\partial y} I_{j n y}(y)+\frac{\mu_{n}}{a}\left[I_{j n}(a, y)-I_{j n}(0, y)\right]+\mu_{l j} I_{j n y} \\
= & \frac{\Delta}{3} \sum_{l=0}^{L} \frac{2 l+1}{2} \sum_{r=1}^{G} c_{r} \alpha k_{r j} P_{l}\left(1+\lambda_{r}-\lambda_{j}\right) P_{l}\left(\mu_{n}\right) \\
& \times \sum_{i=1}^{N} P_{l}\left(\mu_{i}\right) I_{r i y}(x, y) w_{i}
\end{aligned}
$$

For $j=1, \cdots, G$ and $=1, \cdots, N$. Here $I_{j n}(a, y)$ and $I_{j n}(0, y)$ are the angular fluxes at the boundary edges with $x=0, x=a$ and the average angular flux is

$$
I_{j n y}(y)=\frac{1}{a} \int_{0}^{a} I_{j n}(x, y) \mathrm{d} x
$$

Further,

$$
\begin{aligned}
& \mu_{n} \frac{\partial}{\partial x} I_{j n x}(x)+\frac{\eta_{n}}{b}\left[I_{j n}(x, 0)-I_{j n}(x, b)\right]+\mu_{l j} I_{j n x} \\
= & \frac{\Delta}{3} \sum_{l=0}^{L} \frac{2 l+1}{2} \sum_{r=1}^{G} c_{r} \alpha k_{r j} P_{l}\left(1+\lambda_{r}-\lambda_{j}\right) P_{l}\left(\mu_{n}\right) \\
& \times \sum_{i=1}^{N} P_{l}\left(\mu_{i}\right) I_{r i x}(x) w_{i}
\end{aligned}
$$

For $j=1, \cdots, G$ and $=1, \cdots, N$. In Equation (5), $I_{j n}(x, b)$ and $I_{j n}(x, 0)$ are the angular fluxes at the boundary edges with $y=0, y=b$ and the average angular flux is

$$
I_{j n x}(x)=\frac{1}{b} \int_{0}^{b} I_{j n}(x, y) \mathrm{d} y
$$

Application of the $L T S_{N}$ method consists in Laplace transform of the set of $S_{N}$ equations and solving the resulting algebraic equations by matrix diagonalization and subsequent inversion of the transformed angular flux by standard procedures of integral transform theory. The Laplace transform of Equation (3) is

$$
\begin{aligned}
& s \bar{I}_{j n y}(s)+\frac{\mu_{l j}}{\eta_{n}} \bar{I}_{j n y}(s)-\frac{\Delta}{3 \eta_{n}} \sum_{l=1}^{L} \\
& \times \frac{2 l+1}{2} \sum_{r=1}^{G} c_{r} \alpha k_{r j} P_{l}\left(\mu_{n}\right) \sum_{i=1}^{N} P_{l}\left(\mu_{i}\right) \bar{I}_{r i y}(s) w_{i} \\
= & I_{j n y}(0)-\frac{\mu_{n}}{a \eta_{n}}\left[\bar{I}_{j n}(a, s)-\bar{I}_{j n}(0, s)\right]
\end{aligned}
$$

For $j=1, \cdots, G$ and $n=1, \cdots, N$ which can be cast in matrix form,

$$
\left(s I-B_{j n y}\right) \bar{I}_{j n y}(s)=I_{j n y}(0)+\bar{Z}_{(j-1) y}(s)+\bar{S}_{j n y}(s)
$$

Here, $\bar{I}_{j n y}(s)$ are the $N$ componentes of the Laplace transformed angular flux in the y variable and $\bar{I}_{j n y}(0)$ are the respective components of the angular flux at the edge $y=0$. The components have the following forms,

$$
\bar{I}_{j n y}(s)=\left[\bar{I}_{j 1 y}(s), \bar{I}_{j 2 y}(s), \cdots, \bar{I}_{j N y}(s)\right]^{T}
$$

and

$$
\bar{I}_{j n y}(0)=\left[\bar{I}_{j 1 y}(0), \bar{I}_{j 2 y}(0), \cdots, \bar{I}_{j N y}(0)\right]^{T}
$$

The components of the matrix $B_{j n y}$ are given by,

$$
=\left\{\begin{array}{c}
b_{y}(p, q) \\
-\frac{\mu_{l j}}{\eta_{p}}+\frac{\Delta}{3 \eta_{p}} \sum_{l=1}^{L} \frac{2 l+1}{2} c_{j} \alpha k_{j j} P_{l}\left(\mu_{p}\right) P_{l}\left(\mu_{p}\right) w_{p}, p=q \\
\frac{\Delta}{3 \eta_{p}} \sum_{l=0}^{L} \frac{2 l+1}{2} c_{j} \alpha k_{j j} P_{l}\left(\mu_{p}\right) P_{l}\left(\mu_{q}\right) w_{p}, p \neq q
\end{array}\right.
$$

The scattering term is,

$$
\bar{Z}_{(j-1) y}(s)=\sum_{i=1}^{j-1} H_{i j} \bar{I}_{i n y}(s)
$$

with the components of matrix $H_{i j}$ (see the equation below).

The vector $\bar{S}_{j n y}(s)$ has the components,

$$
\bar{S}_{j i y}(s)=-\frac{\mu_{l j}}{a \eta_{i}}\left[\bar{I}_{j i}(a, s)-\bar{I}_{j i}(0, s)\right]
$$

A similar methodology in the $x$ variable leads to the linear algebric system which can be matrix form,

$$
\left(s I-A_{j n x}\right) \bar{I}_{j n x}(s)=I_{j n x}(0)+\bar{Z}_{(j-1) x}(s)+\bar{S}_{j n x}(s)
$$

where $\bar{I}_{j n x}(s)$ has the following form,

$$
\bar{I}_{j n x}(s)=\left[\bar{I}_{j 1 x}(s), \bar{I}_{j 2 x}(s), \cdots, \bar{I}_{j N x}(s)\right]^{T}
$$

The matrix elements of $A_{j n x}$ are,

$$
=\left\{\begin{array}{c}
a_{x}(p, q) \\
-\frac{\mu_{l j}}{\eta_{p}}+\frac{\Delta}{3 \eta_{p}} \sum_{l=0}^{L} \frac{2 l+1}{2} c_{j} \alpha k_{j j} P_{l}\left(\mu_{p}\right) P_{l}\left(\mu_{p}\right) w_{p}, p=q \\
\frac{\Delta}{3 \eta_{p}} \sum_{l=0}^{L} \frac{2 l+1}{2} c_{j} \alpha k_{j j} P_{l}\left(\mu_{p}\right) P_{l}\left(\mu_{q}\right) w_{p}, p \neq q
\end{array}\right.
$$

and the scattering term is

$$
\bar{Z}_{(j-1) x}(s)=\sum_{i=1}^{j-1} H_{i x} \bar{I}_{i n x}(s)
$$

where the matrix elements of $H_{i x}$ are given

$$
\begin{aligned}
& h_{x}(p, q) \\
= & \left\{\begin{array}{l}
\frac{\Delta}{3 \eta_{p}} \sum_{l=0}^{L} \frac{2 l+1}{2} c_{j} \alpha k_{i j} P\left(1+\lambda_{i}-\lambda_{j}\right) P_{l}\left(\mu_{p}\right) P_{l}\left(\mu_{p}\right) w_{p} \\
-\frac{\Delta}{3 \eta_{p}} \sum_{l=0}^{L} \frac{2 l+1}{2} c_{j} \alpha k_{j j} P\left(1+\lambda_{i}-\lambda_{j}\right) P_{l}\left(\mu_{p}\right) P_{l}\left(\mu_{q}\right) w_{p}
\end{array}\right.
\end{aligned}
$$

$$
h_{y}(p, q)=\left\{\begin{array}{l}
\frac{\Delta}{3 \eta_{p}} \sum_{l=0}^{L} \frac{2 l+1}{2} c_{j} \alpha k_{i j} P\left(1+\lambda_{i}-\lambda_{j}\right) P_{l}\left(\mu_{p}\right) P_{l}\left(\mu_{p}\right) w_{p}, p=q \\
-\frac{\Delta}{3 \eta_{p}} \sum_{l=0}^{L} \frac{2 l+1}{2} c_{j} \alpha k_{i j} P\left(1+\lambda_{i}-\lambda_{j}\right) P_{l}\left(\mu_{p}\right) P_{l}\left(\mu_{q}\right) w_{p}, p \neq q
\end{array}\right.
$$


and the vector $\bar{S}_{j n x}(x)$ is

$$
\bar{S}_{j i x}(s)=-\frac{\eta_{i}}{b \mu_{i}}\left[\bar{I}_{j i}(s, b)-\bar{I}_{j i}(s, 0)\right]
$$

The $L T S_{N}$ solution for Equations (8) and (16) are given by

$\bar{I}_{j n y}(s)=\left(s I-B_{j n y}\right)^{(-1)}\left[I_{j n y}(0)+\bar{Z}_{(j-1) y}(s)+\bar{S}_{j n y}(s)\right]$

and

$\bar{I}_{j n x}(0)=\left(s I-A_{j n x}\right)^{-1}\left[I_{j n x}(0)+\bar{Z}_{(j-1) x}(s)+\bar{S}_{j n x}(s)\right]^{T}$

Taking the Laplace inversion by applying the Heaviside expansion yields

$$
\begin{aligned}
I_{j n y}(y)= & \sum_{k=1}^{j n} \beta_{k} e^{s_{k} y} I_{j n y}(0)+Z_{(j-1) y}(y) * \mathcal{L}^{-1}\left\{\left(s I-B_{j n y}\right)^{-1}\right\} \\
& +S_{j n y}(y)^{*} \mathcal{L}^{-1}\left\{\left(s I-B_{j n y}\right)^{-1}\right\}
\end{aligned}
$$

and

$$
\begin{aligned}
I_{j n x}(x)= & \sum_{k=1}^{j n} \beta_{k} e^{s_{k} x} I_{j n y}(0)+Z_{(j-1) x}(x)^{*} \mathcal{L}^{-1}\left\{\left(s I-A_{j n x}\right)^{-1}\right\} \\
& +S_{j n x}(x)^{*} \mathcal{L}^{-1}\left\{\left(s I-A_{j n y}\right)^{-1}\right\}
\end{aligned}
$$

For the fluxes at boundary one may use reasonable approximation [6]

$$
\begin{gathered}
I_{j n}(x, 0)=F_{j n} e^{-s i g n\left(\left(\mu_{n}\right) \Lambda x\right.} \\
I_{j n}(0, y)=G_{j n} e^{-\operatorname{sign}\left(\eta_{n}\right) \Lambda y} \\
I_{j n}(x, b)=O_{j n} e^{-\operatorname{sign}\left(\mu_{n}\right) \Lambda x} \\
I_{j n}(a, y)=P_{j n} e^{-\operatorname{sign}\left(\eta_{n}\right) \Lambda y}
\end{gathered}
$$

Here the signal function follows the usual definition $\operatorname{sign}(\mu)=1$ for $\mu>0$ and $\operatorname{sign}(\mu)=-1$ for $\mu<0$ and $\Lambda$ represents an attenuation parameter, here the macroscopic absorption crosssection.

The generalization of the $L T S_{N}$ nodal solution for a heterogeneous rectangular geometry assuming the Klein-Nishina scattering kernel and a multi-group model may be implemented in a completely analogue procedure. In such a problem the $L T S_{N}$ solution is determined for each subdomain and the integration constants are evaluated upon applying the boundary and interface.

\section{The Two-Dimensional Fokker-Planck Equation Solution for Electrons}

The purpose of radiation transport is to determine how particles move through materials and what effects their propagation have on the material through the mechanisms of deposited energy and charge deposition. The angular flux of electrons in a rectangular domain can be deter- mined by solving the following two-dimensional, time independent electron transport equation, in a rectangle $0 \leq x \leq a$ and $0 \leq y \leq b$, subject to vacuum boundary conditions.

$$
\begin{gathered}
\mu \frac{\partial \psi(x, y, \bar{\Omega}, E)}{\partial x} \\
+\eta \frac{\partial \psi(x, y, \bar{\Omega}, E)}{\partial y}+\sigma_{\mathrm{t}}(E) \psi(x, y, \bar{\Omega}, E) \\
=\int_{4 \pi} \mathrm{d} \bar{\Omega} \sigma_{s}\left(E^{\prime} \rightarrow E, \bar{\Omega}^{\prime} \cdot \bar{\Omega}\right) \psi\left(x, y, \bar{\Omega}^{\prime}, E^{\prime}\right)
\end{gathered}
$$

Here, the angular flux $(x, y, E, \Omega)$, represents the flux of particles at position $(x, y)$, with energy $E$ travelling in direction $\Omega=(\mu, \eta)$. The quantity $\sigma_{s}$ in Equation (25) is the differential scattering cross-section, in this work, we focus on screened Rutherford scattering which is

$$
\sigma_{s}\left(E, \mu_{0}\right)=\frac{\sigma_{t}(E) \eta^{*}\left(\eta^{*}+1\right)}{\pi\left(1+2 \eta^{*}-\mu_{0}^{2}\right)}
$$

where $\eta^{*}>0$ is typically a small constant called the screening parameter parameter is given by

$$
\eta^{*}=\frac{h^{2} Z^{2 / 3}}{4\left(a_{H}\right)^{2}\left(m_{e} v\right)^{2}}
$$

with $Z$ the atomic number of the nucleus, $m v$ the relativistic momentum of the scattered electron and

$$
C=\frac{\bar{h}^{2}}{4 a_{H}^{2}}
$$

which $\bar{h}$ is Planck's constant and $a_{H}$ is the Bohr radius.

We assume that the Fokker-Planck (FP) scattering description is appropriate, so that the transport problem (25) is given by,

$$
\begin{aligned}
& \mu \frac{\partial \psi^{F P}\left(x, y, \bar{\Omega}^{\prime}, E\right)}{\partial x}+\eta \frac{\partial \psi^{F P}\left(x, y, \bar{\Omega}^{\prime}, E\right)}{\partial y} \\
= & \frac{\sigma_{t r}}{2} \frac{\partial}{\partial \mu}\left[\left(1-\mu^{2}\right) \frac{\partial}{\partial \mu}\right] \psi^{F P}\left(x, y, \bar{\Omega}^{\prime}, E\right)
\end{aligned}
$$

Here the coefficient $\sigma_{t r}$ is he transport cross-sectionand defined as,

$$
\sigma_{t r}=2 \pi \int_{-1}^{1} \int_{0}^{1} \sigma_{s}\left(E, \mu_{0}\right)\left(1-\mu_{0}\right) \mathrm{d} \mu_{0} \mathrm{~d} \eta
$$

Upon multiplying Equation (29) by $P_{N}(\mu)$, integrating over $\mu$ and using the recursion formula, as well as general properties of Legendre polynomials leads to the following $P_{N}$ equations

$$
\begin{aligned}
& \frac{n+1}{2 n+1} \frac{\partial}{\partial x} \psi_{n+1}^{F P}(x, y, E)+\frac{n}{2 n+1} \frac{\partial}{\partial x} \psi_{n-1}^{F P}(x, y, E) \\
& +\frac{2 n+1}{n} \frac{\partial}{\partial y} \psi_{n}^{F P}(x, y, E) T_{n}=\frac{\sigma_{t r}}{2}[-n(n+1)] \psi_{n}^{F P}(x, y, E)
\end{aligned}
$$


The angular flux moments in discrete ordinates may be expressed using a quadrature approximation,

$$
\psi^{F P}\left(x, y, \bar{\Omega}^{\prime} E\right)=\sum_{l=0}^{L} \frac{2 l+1}{2} \psi_{n}^{F P}(x, y, E) P_{n}(\mu)
$$

For $n=0, \cdots, N$ with $\psi_{N+1}^{F P}(x, y, E)=0$ in the $P_{N}$ approximation and $T_{n}$ represented by an integral term which can be solved analytically,

$$
T_{n}=\int_{(-1)}^{1} \sqrt{1-\mu^{2}} P_{n}(\mu) P_{(n+1)}(\mu) \mathrm{d} \mu
$$

After applying the Laplace transform in Equation (31) in the spatial variable $x$, we came out with the linear algebric system,

$$
\begin{aligned}
& \frac{n+1}{2 n+1}\left[s \overline{\psi_{n+1}^{F P}}(s, y, E)-\overline{\psi_{n+1}^{F P}}(0, y, E)\right] \\
& +\frac{n}{2 n+1}\left[\overline{\psi_{n-1}^{F P}}(s, y, E)-\overline{\psi_{n-1}^{F P}}(0, y, E)\right] \\
& +\frac{2 n+1}{2} \frac{\partial}{\partial y} \overline{\psi_{n}^{F P}}(s, y, E) T_{n}=\frac{\sigma_{t r}}{2}[-n(n+1)] \overline{\psi_{n}^{F P}}
\end{aligned}
$$

For $n=0, \cdots, N$ and where $\overline{\psi_{n-1}^{F P}}(x, y, E), \overline{\psi_{n}^{F P}}(x, y, E)$ and $\overline{\psi_{n+1}^{F P}}(x, y, E)$ are the transformed angular fluxes in the spatial $x$ variable. Equation (36) can be cast in matrix form,

$$
\begin{aligned}
& A_{n} \overline{\psi_{n}^{F P^{\prime}}}(s, y, E) \\
& +B_{n}(s) \overline{\psi_{n}^{F P}}\left\{(s, y, E)-C_{n} \overline{\psi_{n}^{F P}}(0, y, E)=0\right.
\end{aligned}
$$

where $\overline{\psi_{n}^{F P^{\prime}}}$ are the $N$ components of the derivative of the Laplace transformed angular flux in the $x$ variable.

$$
\overline{\psi_{n}^{F P^{\prime}}}(s, y, E)=\left[\overline{\psi_{n}^{F P^{\prime}}}(s, y, E), \cdots, \overline{\psi_{n}^{F P^{\prime}}}(s, y, E)\right]^{T}
$$

The components of matrices $A_{N}, B_{N}(s)$ and $C_{N}$ are for $i \in\{1,2, \cdots, N\}$, respectively

$$
\begin{aligned}
& a_{i j}=\left\{\begin{array}{l}
(2 i+1)^{2} T_{i}, i=j \\
0, i \neq j
\end{array}\right. \\
& b_{i j}=\left\{\begin{array}{l}
i(i+1)(2 i+1) \sigma_{t r}, i=j \\
2 i s, i=|j-1| \\
0, \text { otherwise }
\end{array}\right. \\
& c_{i j}= \begin{cases}2 i, & i=|j-1| \\
0, & \text { otherwise }\end{cases}
\end{aligned}
$$

The solution of Equation (35) is

$$
\begin{aligned}
\overline{\psi_{n}^{F P}}(s, y, E)= & c_{1}(s) e^{-\left(B_{n}(s) A_{n}^{-1}\right)} \\
& +C_{n}\left(B_{n}(s)\right)^{-1} \psi_{n}^{F P}(0, y, E)
\end{aligned}
$$

where $c_{1}(s)$ is an arbitrary constant determined by ap- plying the boundary and interface conditions. Due to the linear character of the inverse Laplace transform operator, the solution is composed by

$$
\begin{aligned}
\psi_{n}^{F P}(x, y, E)= & \mathcal{L}^{-1}\left\{c_{1}(s) e^{-\left(B_{n}(s) A_{n}^{-1}\right)}\right\} \\
& +C_{n} \cdot \mathcal{L}^{-1}\left\{\left(B_{n}(s)\right)^{-1}\right\} \cdot \psi_{n}^{F P}(0, y, E)
\end{aligned}
$$

\section{Numerical Results}

In the following we apply the closed-form formulation to the build-up factor and absorbed energy for photons and electrons in the Compton energy range and in Cartesian geometry. Moreover, we the one-dimensional, two-dimensional problem for photons and two-dimensional problem for photons and electrons. To this end we evaluate the exposure build-up factor, considering a scalar flux of the photons with energy $1 \mathrm{MeV}$, incident in a multi-layered slab with two regions, composed of water and lead, water and iron, lead and iron. Assuming that the kernel is described by the Klein-Nishina differential scattering crosssection and the energy variables may be simplified in form of a multi-group model in the wavelength (energy) variable.

Here the exposure build-up factor is defined as the sum of the product of the attenuation coefficient of the air with the scalar flux for all photons, including the incident flux, divided by the attenuation coefficient of the air for the incident flux multiplied by the incident scalar flux. The numerical results for three problems are shown in Table 1 for water and lead, in Table 2 for water and iron and in Table 3 for lead and iron. In Table 1 we present the $L T S_{N}$ numerical simulations for the exposure build-up factor and comparisons with results from the $E G S_{4}$ code [7] generated for the one-group model. We consider a multi-layered slab with two regions, composed of water $\left(\mu_{i j}=0.0707 \mathrm{~cm}^{2} / \mathrm{g}\right.$ and $\left.1.0 \mathrm{mpf}\right)$ and lead $\left(\mu_{i j}=0.06848\right.$ $\mathrm{cm}^{2} / \mathrm{g}$ and depth in multiples of the mean free path, 4.0, 5.0, 10.0, 20.0, 30.0 and $40.0 \mathrm{mfp}$ ) together with the afore mentioned vacuum boundary conditions.

Table 1. Numerical exposure buildup factor simulations for a multilayered slab composed of water $(1.0 \mathrm{mfp})$ and lead.

\begin{tabular}{ccc}
\hline Iron (mfp) & $L_{T S_{16}}$ & $E G S_{4}$ \\
\hline 4.0 & 2.30 & 2.31 \\
5.0 & 2.07 & 2.08 \\
10.0 & 3.57 & 3.59 \\
20.0 & 5.29 & 5.31 \\
30.0 & 6.77 & 6.79 \\
40.0 & 8.26 & 8.27 \\
\hline
\end{tabular}


Table 2. Numerical exposure buildup factor simulations for a multilayered slab composed of water $(1.0 \mathrm{mfp})$ and iron.

\begin{tabular}{ccc}
\hline Iron (mfp) & LTS $_{16}$ & $E G S_{4}$ \\
\hline 4.0 & 4.99 & 5.01 \\
5.0 & 6.21 & 6.23 \\
10.0 & 13.9 & 13.9 \\
20.0 & 36.3 & 36.3 \\
30.0 & 67.6 & 67.5 \\
40.0 & 101.0 & 101.0 \\
\hline
\end{tabular}

Table 3. Numerical exposure buildup factor simulations for a multilayered slab composed of lead (1.0 $\mathrm{mfp})$ and iron.

\begin{tabular}{ccc}
\hline Iron (mfp) & $L T S_{16}$ & $E G S_{4}$ \\
\hline 4.0 & 4.87 & 4.86 \\
5.0 & 6.34 & 6.28 \\
10.0 & 15.4 & 15.3 \\
20.0 & 41.5 & 41.4 \\
30.0 & 78.4 & 78.3 \\
40.0 & 118.0 & 117.0 \\
\hline
\end{tabular}

In Table 2 we present the $L T S_{N}$ numerical simulations for the exposure build-up factor and comparisons with the results from $E G S_{4}$ generated for the one-group model. We consider a multi-layered slab with two regions, composed of water $\left(\mu_{i j}=0.0707 \mathrm{~cm}^{2} / \mathrm{g}\right.$ with depth of 1.0 mpf) and iron $\left(\mu_{i j}=0.0596 \mathrm{~cm}^{2} / \mathrm{g}\right.$ with depth $4.0,5.0$, $10.0,20.0,30.0$ and $40.0 \mathrm{mfp}$ ) and vacuum boundary conditions.

From the analysis of the results encountered in Tables 1-3, one realizes a fairly good agreement between the $L T S_{16}$ and $E G S_{4}$ results. The numerical convergence of the $L T S_{N}$ results showed for increasing $N$ a coincidence of six significant digits for $N=14$ and $N=16$. For two-dimensional problems for photons and electrons, we applied the $L T S_{N}$ method, for $N=8$, in the transport equation for photons and used $N=9$ in the $P_{N}$ approximation for the angular variable of the Fokker-Planck equation for electrons. We considered homogeneous rectangular geometries composed of water liquid $(Z / A=0.55508$, $\rho=1 \mathrm{~g} / \mathrm{cm}^{2}$ ), soft tissue (ICRU44, $Z / A=0.54996, \rho=$ $1.06 \mathrm{~g} / \mathrm{cm}^{2}$ ) and cortical bone (ICRU44, Z/A $=0.51478, \rho$ $\left.=1.92 \mathrm{~g} / \mathrm{cm}^{2}\right)$. Further, we assumed a mono-energetic $(\mathrm{E}$ $=1.25 \mathrm{MeV}$ ) and monodirectional photon beam incident on the edge of a rectangle with dimension $20 \mathrm{~cm} \times 20 \mathrm{~cm}$ and vacuum boundary conditions. The incoming photons were tracked until their whole energy was deposited and/ or they left the domain of interest. In this problem also the energy deposited by the secondary electrons, generated by the Compton Effect, were considered. Other possible effects, however with small or spurious contributions were not taken into account. The numerical results encountered for absorbed energy in the domain were compared with simulations obtained with the program Geant 4 v8 [8], using the low energy libraries and are presented in Tables 4 and 5.

The numerical convergence of the $L T S_{N}$ results showed for increasing $N$ a coincidence of three significant digits for $N=4,6$ and 8 . Notice, the coincidence of four significant digits for the $P_{N}$ approximation with $N=7$ and 9 . These results were obtained in the homogeneous domain with dimension $20 \mathrm{~cm} \times 20 \mathrm{~cm}$ that was composed of water. In Table 4 we present the $L T S_{8}$ nodal numerical simulations for the absorbed energy induced by photons incident on a homogeneous rectangular domain, that is composed of a variety of different materials. In Table 5 we present numerical simulations for absorbed energy by the $P_{9}$ approximation, due to free electrons, arising from Compton scattering in a homogeneous rectangular domain composed of different materials. These results were compared with simulations obtained by Geant 4 .

In spite of the fact, that two different methods were used to simulate energy deposition, the Monte Carlo method with Geant 4 and our closed form solution the results in Tables $\mathbf{4}$ and $\mathbf{5}$ show a fairly good agreement. From the results, we notice that the maximum discrepancy found is $3.4 \%$ in the simulations for photons and $8.3 \%$ for electrons. The difference of our numerical results in comparison to the Geant simulations, that contain a catalogue of processes, demonstrate that other effects shall be taken into account. As the material density increases, the

Table 4. Absorved energy (KeV/photon emitted from the source) by the photons incident in a homogeneous domain dimension $20 \mathrm{~cm} \times 20 \mathrm{~cm}$, composed of materials different.

\begin{tabular}{cccc}
\hline Domain composition & LTS & Geant4 & Discrepancy (\%) \\
\hline Water liquid & 0.00457 & 0.00468 & 2.3 \\
Soft tissue & 0.00531 & 0.00542 & 2.0 \\
Cortical bone & 0.0987 & 0.09487 & 3.4 \\
\hline
\end{tabular}

Table 5. Absorved energy (KeV/photon emitted from the source) by the free electrons in a homogeneous domain dimension $20 \mathrm{~cm} \times 20 \mathrm{~cm}$, composed of materials different.

\begin{tabular}{cccc}
\hline Domain composition & LTS & Geant4 & Discrepancy (\%) \\
\hline Water liquid & 0.03379 & 0.03609 & 6.4 \\
Soft tissue & 0.03317 & 0.03542 & 6.4 \\
Cortical bone & 0.79284 & 0.86380 & 8.3 \\
\hline
\end{tabular}


number of interactions increases as well as the possibility of other production processes involving secondary electrons, responsible for more than $86 \%$ of the total energy absorbed in domain.

\section{Conclusion}

Concluding, we were successful in determinig the $L T S_{N}$ solution in closed form forenergy deposition induced by photons in Cartesian geometry. From the solution we obtained the buildup factor and absorbed energy, for photons and electrons, in the Compton energy range. It is worth mentioning, that the $L T S_{N}$ procedure maintains an analytical character of the solution and the unique approximation made was in the leakage angular flux at the boundary. The $P_{N}$ solution of the Fokker-Planck equation remains analytical in the sense that no approximation is made along its derivation from $P_{N}$ equations, except for the truncation. Finally, a variety of additional numerical experiments have shown us that the presented method is robust for problems of the considered transport equation type.

\section{Acknowledgements}

The authors are gratefully to CNPq (Conselho Nacional de Desenvolvimento Científico e Tecnológico) for the partial financial support of this work. A special acknowledgment for the project INCT (Instituto Nacional de Ciencia e Tecnologia-Reatores Nucleares Inovadores) for financial support.

\section{REFERENCES}

[1] C. F. Segatto, M. T. Vilhena and R. P. Pazos, "On the Convergence of the Spherical Harmonics Approximations," $\mathrm{Nu}$ clear Science and Engineering, Vol. 134, No. 1, 2000, pp. 114-119.

[2] M. T. Vilhena, C. F. Segatto and L. B. Barichello, "A Particular Solution for the SN Radiative Transfer Problems," Journal of Quantitative Spectroscopy and Radiative Transfer, Vol. 53, No. 4, 1995, pp. 467-469. doi:10.1016/0022-4073(95)90020-9
[3] C. Borges and E. W. Larsen, "The Transversely Integrated Scalar Flux of a Narrowly Focused Particle Beam," SIAM Journal on Applied Mathematics, Vol. 55, No. 1, 1995, pp. 1-22.

[4] C. Borges and E. W. Larsen, "On the Accuracy of the Fokker-Planck and Fermi Pencil Beam Equation for Charged Particle Transport," Medical Physics, Vol. 23, No. 10, 1996, pp. 1749-1759. doi:10.1118/1.597832

[5] B. D. A. Rodriguez, M. T. Vilhena, V. Borges and G. Hoff, "A Closed Form Solution for the Two-Dimensional Fokker-Planck Equation for Electron Transport in the Range of Compton Effect," Annals of Nuclear Energy, Vol. 35, No. 5, 2008, pp. 958-962.

doi:10.1016/j.anucene.2007.09.002

[6] B. D. A. Rodriguez, M. T. Vilhena and V. Borges, “A Solution for the Two-Dimensional Transport Equation for Photons and Electrons in a Rectangular Domain by the Laplace Transform Technique," International Journal of Nuclear Energy Science and Technology, Vol. 5, No. 1, 2010, pp. 25-40. doi:10.1504/IJNEST.2010.030304

[7] H. Hirayama and K. Shin, "Application of the $\mathrm{EGS}_{4}$ Monte Carlo Code to a Study of Multilayer Gamma-Ray Exposure Buildup Factors," Journal of Nuclear Science and Technology, Vol. 35, No. 11, p. 816. doi:10.3327/jnst.35.816

[8] D. H. Wright, "Physics Reference Manual," 2001. http://cern.ch/geant4

[9] G. C. Pomraning, "Flux-Limited Diffusion and FokkerPlanck Equations," Nuclear Science and Engineering, Vol. 85, No. 2, 1983, p. 116.

[10] J. Wood, "Computational Methods in Reactor Shielding," Pergamon Press, Oxford, 1982.

[11] S. Agostinelli, et al., "Geant4: A Simulation Toolkit," Nuclear Instruments and Methods in Physics Research A, Vol. 506, No. 3, 2003, pp. 250-303.

[12] DOORS 3.1, "One, Two and Three Dimensional Discrete Ordinates Neutron Photon Transport Code System," Radiation Safety Information Computational Center (RSICC), Code Package CCC-650, Oak Ridge, Tennessee, 1996.

[13] B. D. A. Rodriguez, M. T. Vilhena and V. Borges, "The Determination of the Exposure Buildup Factor Formulation in a Slab Using the LTS ${ }_{\mathrm{N}}$ Method," Kerntechnik, Vol. 71, No. 4, 2006, pp. 182-184. 